\title{
Long-term outcome after laparoscopic myotomy for achalasia
}

\author{
Pradheep Krishnamohan, MD, ${ }^{\mathrm{a}}$ Mark S. Allen, MD, ${ }^{\mathrm{a}} \mathrm{K}$. Robert Shen, MD, ${ }^{\mathrm{a}}$ Dennis A. Wigle, MD, ${ }^{\mathrm{a}}$ \\ Francis C. Nichols III, MD, ${ }^{a}$ Stephen D. Cassivi, MD, ${ }^{a}$ William S. Harmsen, MS, ${ }^{b}$ and Claude Deschamps, MD $^{a}$
}

Objectives: Achalasia has a variety of therapeutic options. We sought to determine the long-term outcome of laparoscopic myotomy in a large group of patients.

\begin{abstract}
Methods: We conducted a retrospective record review of 500 patients who underwent laparoscopic myotomy and follow-up with a standardized dysphagia questionnaire.

Results: Between April 1998 and June 2011, 276 men and 224 women underwent a laparoscopic myotomy. Their median age was 51 years (range, 8-89 years). Preoperative symptoms included dysphagia in 472 patients (94.4\%), regurgitation in 382 patients $(76.4 \%)$, heartburn in 203 patients $(40.6 \%)$, chest pain in $168(33.6 \%)$ patients, and weight loss (median, $10 \mathrm{~kg}$ ) in 264 patients (52.8\%). Prior therapy (dilation in 239, botulinum toxin type $\mathrm{A}$ in 135 , or myotomy in 30 ) had been performed in 303 patients $(60.6 \%)$. Preoperative manometry was available in 413 patients $(82.6 \%)$ and revealed a median lower esophageal sphincter pressure of $31 \mathrm{~mm} \mathrm{Hg}$ (range, $0-50 \mathrm{~mm} \mathrm{Hg}$ ), absent peristalsis in $97.6 \%$, and no lower esophageal sphincter relaxation in $75.1 \%$. A laparoscopic myotomy with partial fundoplication (Toupet $\mathrm{n}=268$, Dor $\mathrm{n}=209$ ) was done in 477 patients $(95.4 \%)$ and myotomy only in 23 patients $(4.6 \%)$. Conversion to open was necessary in 16 patients $(3.2 \%)$. There were $52(10.4 \%)$ esophageal perforations-all repaired during the myotomy-and $26(5.2 \%)$ other complications, including bleeding, atrial fibrillation, and pneumonia. There was no operative mortality. Median length of stay was 2 days (range, 1-20 days). Dysphagia questionnaires were returned by $48.2 \%$ of patients (241 out of 500) a median of 77.5 months after myotomy (range, 15-176 months). Eighty patients $(32.2 \%)$ reported no problems with swallowing at the time of the survey. Of 160 patients who had swallowing problems, 40 patients $(22.2 \%$ ) classified the problem as mild or less, 98 patients classified the problem as moderate $(70.0 \%)$, and 22 patients classified the problem as severe $(15.7 \%)$. The question concerning frequency of swallowing problems was answered by 160 patients and occurred once a week or less in 70 patients (43.8\%), several times a week in 41 patients (25.6\%), and at least daily in 49 patients (30.6\%). Analysis of all 241 patients who returned questionnaires revealed that 170 out of $241(70.5 \%)$ reported heartburn, 89 out of $241(36.9 \%)$ reported regurgitation, and 159 out of $241(66.0 \%)$ had taken antacids or histamine 2 blockers or proton pump inhibitors within the past 30 days of the survey. The only factor affecting outcome was age; patients older than age 65 years had a better chance of having no difficulty swallowing at follow-up $(P=.0174)$. Sex, prior therapy, Toupet versus Dor, conversion to open, or esophageal mucosal perforation did not affect long-term swallowing outcome.
\end{abstract}

Conclusions: Laparoscopic myotomy with partial fundoplication is a safe operation. The long-term outcome is extremely effective and approximately one-third of patients have no evidence of persistent symptoms at follow-up. Furthermore, those who have persistent symptoms rarely have severe or very frequent complaints. Patients older than age 65 years tend to have a better outcome. (J Thorac Cardiovasc Surg 2014;147:730-7)

Achalasia is a disease characterized by failure of the lower esophageal sphincter (LES) to relax and loss of

\footnotetext{
From the Division of General Thoracic Surgery, ${ }^{\text {a }}$ Department of Surgery, and the Division of Biomedical Statistics and Informatics, ${ }^{b}$ Department of Health Sciences Research, Mayo Clinic, Rochester, Minn.

Disclosures: Authors have nothing to disclose with regard to commercial support.

Read at the 93rd Annual Meeting of The American Association for Thoracic Surgery, Minneapolis, Minnesota, May 4-8, 2013.

Received for publication May 6, 2013; revisions received Sept 10, 2013; accepted for publication Sept 30, 2013; available ahead of print Nov 18, 2013.

Address for reprints: Mark S. Allen, MD, Division of General Thoracic Surgery, Mayo Clinic, 200 First St, SW, Rochester, MN 55905 (E-mail: allen.mark@ mayo.edu).

0022-5223/\$36.00

Copyright (c) 2014 by The American Association for Thoracic Surgery

http://dx.doi.org/10.1016/j.jtcvs.2013.09.063
}

esophageal peristalsis. The classic treatment is to render the LES incompetent to relieve the obstructive symptoms and prevent massive esophageal dilation. Destruction of the LES can lead to significant symptoms from reflux esophagitis. Laparoscopic techniques are now used to perform a surgical myotomy and perform a partial fundoplication to treat patients with achalasia. We have employed this technique since the mid-1990s as the main surgical therapeutic intervention in patients with achalasia. We sought to examine the long-term outcome of the procedure by using a standardized 30-day dysphagia questionnaire on patients who have undergone laparoscopic treatment for achalasia at our institution. 


\section{Abbreviations and Acronyms \\ $\mathrm{H}_{2}$ = histamine 2 \\ LES $=$ lower esophageal sphincter \\ PPI $=$ proton pump inhibitor}

\section{MATERIALS AND METHODS}

Mayo Foundation Institutional Review Board approved this study. All patients who underwent surgical treatment for achalasia at the Mayo Clinic in Rochester, Minnesota, between April 1998 and June 2011 were reviewed. Eligible patients included those with a diagnosis of achalasia who underwent an attempted laparoscopic myotomy with or without a fundoplication. Patients who underwent a thoracotomy for a long myotomy or underwent an esophagectomy for achalasia were excluded. Medical records were reviewed for demographic information, medical history, radiographic findings, surgical approach, and procedural and postoperative complications. A standardized 30-day dysphagia questionnaire was sent to all patients during the follow-up period. ${ }^{1}$ Postoperative complications included those that occurred within 30 days of the procedure or during the same hospitalization. Similarly, operative mortality included patients who died within the first 30 days after surgery or during the same hospital admission. Factors affecting functional results were analyzed by univariate and multivariate analysis. Descriptive statistics for categorical variables are reported as frequency and percentage, and continuous variables are reported as mean \pm standard deviation or median (range), as appropriate. All statistical tests were 2-sided.

\section{RESULTS}

There were 500 patients-276 men and 224 womenwho underwent surgical myotomy for achalasia during the study period (Table 1). The median age was 51 years (range, 8-89 years). Preoperative symptoms were present in 472 patients $(94.4 \%)$. Weight loss before the surgical procedure had occurred in 264 patients $(52.8 \%)$. Median weight loss was $10 \mathrm{~kg}$ (range, $1-80 \mathrm{~kg}$ ).

Preoperative esophageal motility data was available in 413 patients $(82.6 \%)$ and showed no peristalsis in 403 patients and only minimal contractions in 10 patients. LES pressure measurements were available in 367 patients. The median LES was $31 \mathrm{~mm} \mathrm{Hg}$ (range, 4-150 mm Hg) and was $>25 \mathrm{~mm} \mathrm{Hg}$ in 233 patients. There was data on LES relaxation in 412 patients; 320 patients showed no LES relaxation and 93 had some. Prior interventional therapy had been performed in 303 of patients $(60.6 \%)$. The interventions included dilations in 239 patients $(47.8 \%)$, botulinum toxin type A injections in 135 patients $(27.0 \%)$, and prior myotomy in 30 patients $(6.0 \%)$. The 30 prior myotomies were performed a median of 4.7 years before (range, 3.8 months-50 years). The reoperation was a laparoscopic myotomy with Toupet fundoplication in 4 patients, with Dor fundoplication in 9 patients, and with no fundoplication in 18 patients. Only 2 of these patients required conversion to an open procedure.

The operative procedure was laparoscopic myotomy with Toupet fundoplication in 268 patients $(53.6 \%)$, laparoscopic myotomy with Dor fundoplication in 209 patients $(41.8 \%)$, and laparoscopic myotomy only in 23 patients $(4.6 \%)$. The choice of a Dor or Toupet fundoplication was based on surgeon's choice. The myotomy typically went as high as possible using a laparoscopic approach (typically above the inferior pulmonary vein) and was carried onto the stomach for $2 \mathrm{~cm}$. Conversion to an open procedure was necessary in 16 patients $(3.2 \%)$. Reasons for conversion were to repair a breach of the esophageal mucosa in 7 patients, to control bleeding in 4 patients, intra-abdominal adhesions in 3 patients, and inability to find the correct myotomy plane in 2 patients. The rate of conversion did not vary significantly during the 14 years of the study and ranged from 1 to 3 conversions per year. The median length of stay after surgery was 2 days (range, 1-20 days); 374 patients (74.8\%) stayed 2 days or fewer. Median length of stay for patients that required an open procedure was 5 days (range, 3-10 days). There were no operative mortalities. Complications occurred in 18 patients $(3.6 \%)$ and included urinary retention in 4 patients; ileus in 3 patients; atrial fibrillation in 3 patients; bowel perforation requiring subsequent surgery in 2 patients (1 was from a esophageal perforation at the time of myotomy that subsequently leaked and was repaired with a Belsey procedure, the other was from a unrecognized small bowel perforation created while taking down adhesions); pneumonia in 2 patients; and urinary infection, respiratory failure, diarrhea, leg cellulitis, herpes zoster, and esophageal obstruction from diaphragmatic constriction requiring reoperation in 1 patient each. The overall rate of esophageal perforation at the time of myotomy was $10.4 \%$ (52 out of 500 patients) and was affected by prior therapy. Of the 30 patients that had undergone a prior myotomy and 7 patients $(23.3 \%)$ had a mucosal perforation at the time of redo laparoscopic myotomy; however, having a dilation or botulinum toxin type A injection did not significantly affect the rate of esophageal perforation at the time of laparoscopic myotomy ( $11.7 \%$ and $10.4 \%$, respectfully).

Dysphagia questionnaires were sent to all 500 patients; $241(48 \%)$ returned a completed survey. There was no response from the survey from 164 patients $(33 \%)$, the survey was returned unopened from 64 patients $(13 \%)$, notification of the patient's death occurred in 10 patients $(2 \%)$, and 21 patients $(4 \%)$ declined to participate in the survey. There was no statistically significant difference in the median age or sex of patients who returned the survey and those who did not; however, there was a difference in the median time from surgery to the return of the survey in those who responded to the survey and those who did not respond (6.4 years between surgery and the survey for responders vs 7.4 years for nonresponders; $P=.0033$ ). Completed surveys were returned a median of 77.5 months after surgery (range, 15 months- 14.7 years).

There were 80 out of 241 patients (33.2\%) who reported they had no swallowing problems on the follow-up 
TABLE 1. Demographic and clinical data of patients who underwent laparoscopic myotomy

\begin{tabular}{|c|c|c|c|}
\hline Variable & $\begin{array}{c}\text { Overall } \\
(\mathbf{N}=\mathbf{5 0 0})\end{array}$ & $\begin{array}{c}\text { Answered } \\
\text { questionnaire } \\
(\mathbf{n}=\mathbf{2 4 1} ; \mathbf{4 8 . 2} \%) \\
\end{array}$ & $\begin{array}{c}\text { Did not answer } \\
\text { questionnaire } \\
(\mathbf{n}=\mathbf{2 5 9} ; \mathbf{5 1 . 8} \%)\end{array}$ \\
\hline $\begin{array}{l}\text { Age, y (median } \\
\text { [range]) }\end{array}$ & $51(8-89)$ & $53(11-88)$ & $48(8-89)$ \\
\hline \multicolumn{4}{|l|}{ Sex, n } \\
\hline Men & $276(55)$ & 137 (56.8) & $139(53.7)$ \\
\hline Women & $224(45)$ & $104(43.2)$ & $120(46.3)$ \\
\hline \multicolumn{4}{|l|}{ Symptoms } \\
\hline \multicolumn{4}{|l|}{ Dysphagia } \\
\hline Solids & $295(59)$ & $144(59.8)$ & $151(58.3)$ \\
\hline Liquids & $19(3.8)$ & $9(3.7)$ & $10(3.9)$ \\
\hline Solids and liquids & 158 & $74(30.7)$ & $84(32.4)$ \\
\hline Regurgitation & $382(76.4)$ & $179(74.3)$ & 203 (78.4) \\
\hline Heartburn & $203(40.6)$ & $102(42.3)$ & $101(39.0)$ \\
\hline Chest pain & $168(33.6)$ & $83(34.4)$ & $85(32.8)$ \\
\hline Manometry & $413(82.6)$ & $186(77.2)$ & 227 (87.6) \\
\hline Absent peristalsis & $403(80.6)$ & $225(93.4)$ & $178(68.7)$ \\
\hline $\begin{array}{l}\text { LES pressure, } \\
\text { mm Hg (median } \\
\text { [range]) }\end{array}$ & $31(4-150)$ & $34(4-150)$ & $31(6-116)$ \\
\hline $\begin{array}{l}\text { Absent LES } \\
\text { relaxation }\end{array}$ & $320(64.0)$ & $160(66.4)$ & $160(61.8)$ \\
\hline Prior therapy & $303(60.6)$ & $144(59.8)$ & $159(61.4)$ \\
\hline Dilation & $239(47.8)$ & $111(46.1)$ & $128(49.4)$ \\
\hline $\begin{array}{l}\text { botulinum toxin } \\
\text { type A injection }\end{array}$ & $135(27.0)$ & $59(24.5)$ & $76(29.3)$ \\
\hline Surgical myotomy & $30(6.0)$ & $15(6.2)$ & $15(5.8)$ \\
\hline
\end{tabular}

Values are presented as number (\%) unless otherwise specified. LES, Lower esophageal sphincter.

dysphagia questionnaire. Of 161 patients $(66.8 \%)$ who reported problems swallowing, when asked to rate the severity of their swallowing difficulty over the past 30 days, 9 patients $(5.7 \%)$ responded that it did not bother them at all, $29(18.4 \%)$ described it as mild, or "can be ignored if I don't think about it," 98 (62.0\%) described it as moderate, "cannot be ignored, but does not affect my lifestyle," 17 (10.8\%) described it as severe, "affects my lifestyle," and $5(3.2 \%)$ described it as very severe, "markedly affects my lifestyle." Three patients did not answer the question. Patients were asked to rate their swallowing trouble on a scale of 1 (not severe at all) to 10 (very severe). The median response was 4 (range, 1-10) (Figure 1). When asked how often they had difficulty swallowing in the past 30 days, $68(43 \%)$ answered once a week or less, $41(26 \%)$ answered several times a week, $24(15 \%)$ answered daily, and $25(16 \%)$ answered with each meal. Of those with swallowing problems, 50 patients $(31.3 \%)$ had difficulty with liquids and 149 patients (93.1\%) had difficulty with solid food. The types of solid food that patients had difficulty with are shown in Table 2.

There were 98 patients who had follow-up of 5 years or less and 37 patients $(46.3 \%)$ had no trouble swallowing. Of the 61 who had swallowing difficulty and followed for 5 years or fewer, only $7(7.1 \%)$ patients had a severity $>5$. There were 143 followed for $>5$ years; $43(53.8 \%)$ had no trouble swallowing; however, in this group with longer follow-up there were $30(16.1 \%)$ who stated their severity of swallowing difficulty was $>5$ (Figure 2). Neither of these differences was statistically significant $(P=.23$ and .22 , respectively).

There were 113 patients who had all 3 classic findings on manometry for achalasia: LES $>20 \mathrm{~mm} \mathrm{Hg}$, no peristalsis, and no LES relaxation. In this group 41 patients $(36.3 \%)$ had no difficulty swallowing at follow-up, but there were 19 patients $(16.8 \%)$ who rated their swallowing difficulty $>5$. There were 77 patients who had at least 1 of the classic manometric findings missing from their preoperative

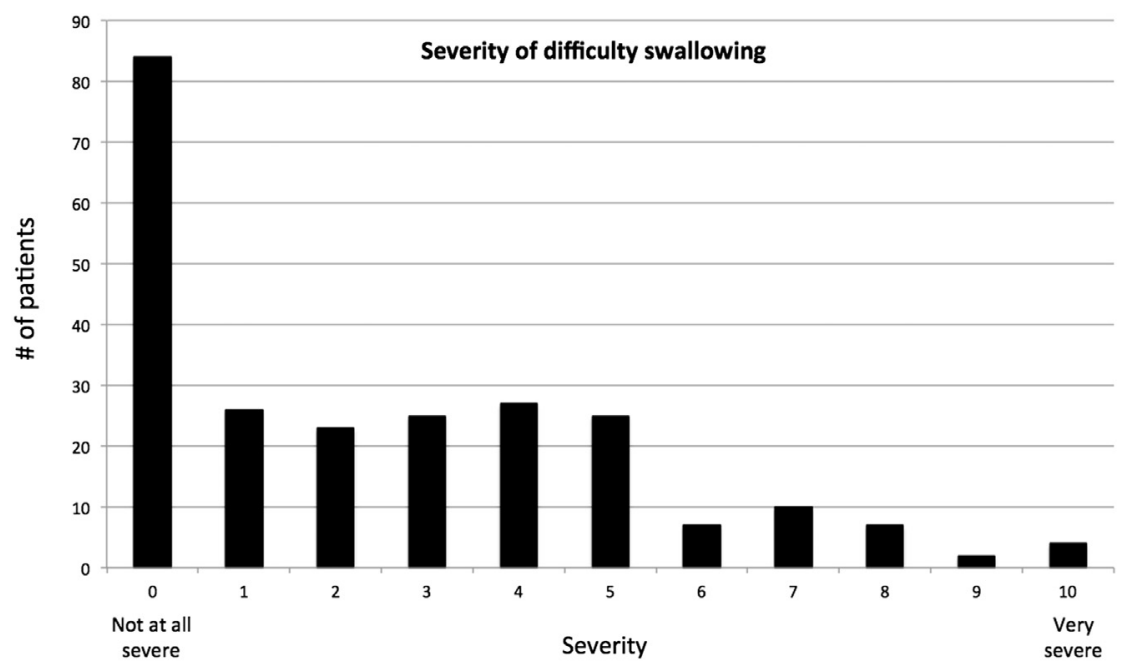

FIGURE 1. Rating of severity of swallowing difficulty after laparoscopic myotomy. 
TABLE 2. Patients that had trouble swallowing foods

\begin{tabular}{lrc}
\hline \multicolumn{1}{c}{ Type of food } & n & $\%$ \\
\hline Oatmeal (or other foods, like grits, cream of wheat, or rice) & 21 & 13.3 \\
Banana (or other foods, like pudding or gelatin dessert) & 26 & 16.5 \\
Apple (or other foods with fiber, such as celery) & 80 & 50.6 \\
Ground meat (like hamburger or ground turkey) & 71 & 44.7 \\
Bread (or other foods, like cake, donuts, or muffins) & 114 & 71.7 \\
Meat (fibrous meats, like steak or chicken) & 110 & 68.8 \\
\hline
\end{tabular}

manometry. In this group, 24 patients $(31.2 \%)$ had no swallowing difficulty at follow-up, but there were only $5(6.5 \%)$ who rated their swallowing difficulty $>5$ (Figure 3). Neither of these differences was statistically significant $(P=.47$ and .95 , respectively).

Of 30 patients $(6.0 \%)$ who had undergone a prior myotomy, $15(50 \%)$ returned the questionnaire. Only 2 patients $(13.3 \%)$ had no swallowing problems at follow-up. On a severity scale of 1 to 10 patients who had swallowing problems reported that the severity was a median of 5 (range, 2-8). When asked how often they had difficulty swallowing in the past 30 days, $60 \%$ stated they had difficulty at least once a week.

Among patients who responded to the questionnaire, 170 $(70.5 \%)$ said they had experienced heartburn during the past 30 days. Of those that experienced heartburn in the past 30 days, it occurred once a month or less in $41 \%$, about once a week in $32 \%$, and several times a week or daily in $28 \%$. Regurgitation occurred in 89 patients $(36.9 \%)$ but only $30(33.7 \%)$ of these complained of regurgitation at least once a week. There were 159 survey responders $(66.0 \%)$ who were taking antacids, histamine 2 receptor $\left(\mathrm{H}_{2}\right)$ blockers, or proton pump inhibitors (PPIs) at followup (antacids, 114 [47.3\%]; $\mathrm{H}_{2}$ blockers, 38 [15.8\%]; and PPIs, $96[39.8 \%])$. There were 23 patients who had myotomy only, without fundoplication. Eleven of these 23 patients returned a questionnaire and 9 patients $(81.8 \%)$ still had dysphagia and 7 patients $(63.6 \%)$ had reflux symptoms, 5 of them at least weekly.
Factors affecting whether or not patients stated they had any swallowing problems or not during the past 30 days at follow-up were examined. Only age $\geq 65$ years affected this outcome variable. For patients aged 65 years or older, the odds ratio (OR) was 2.1 (95\% confidence interval [CI], $1.10-4.02 ; P=.0252$ ) for this older group to state they did not have any swallowing problems in the past 30 days. Other factors, including sex, previous dilation, botulinum toxin type A injection, or myotomy, Toupet versus Dor, conversion to open procedure, and esophageal perforation did not affect the outcome. Similarly, patients aged 65 years and older were less likely to experience heartburn or regurgitation after a myotomy (OR, 2.30; 95\% CI, 1.18-4.48; $P=.0146)$. Sex, prior dilation, botulinum toxin type A, or myotomy, Toupet versus Dor, conversion to open lobectomy, and esophageal perforation did not affect whether or not patients experienced heartburn or regurgitation at follow-up. We did find that women were less likely than men to be taking antacids, $\mathrm{H}_{2}$ blockers, or PPIs at follow-up but this was not statistically significant (OR, 0.52; 95\% CI, 0.30-0.91; $P=.02$ ). Age, prior dilation, botulinum toxin type A, or myotomy, Toupet versus Dor, conversion to open lobectomy, and esophageal perforation did not affect whether or not patients were taking antacids, $\mathrm{H}_{2}$ blockers, or PPIs at follow-up.

\section{DISCUSSION}

Achalasia has a variety of treatment options, including endoscopic injection of botulinum toxin type A, balloon dilation, and surgical myotomy. In this review of our experience with laparoscopic myotomy for achalasia, we tried to examine the long-term outcome of the procedure. By using a standardized questionnaire we found that the therapy is extremely effective and approximately one-third of patients have no esophageal symptoms at follow-up. In patients who did have symptoms they were relatively infrequent.

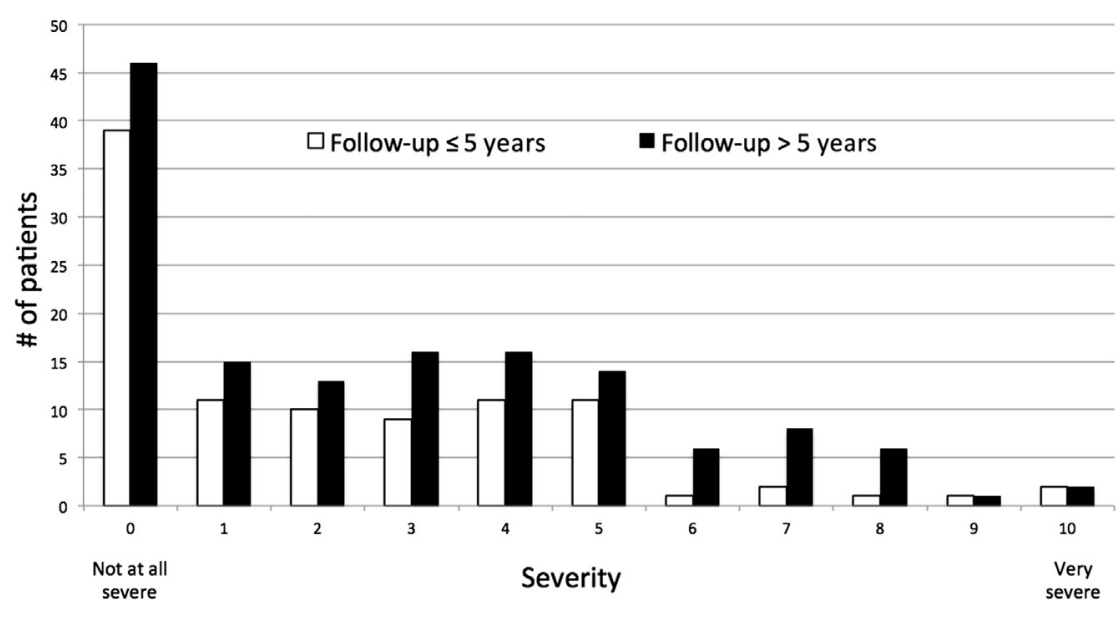

FIGURE 2. Rating of severity of swallowing difficulty according to length of follow-up. 


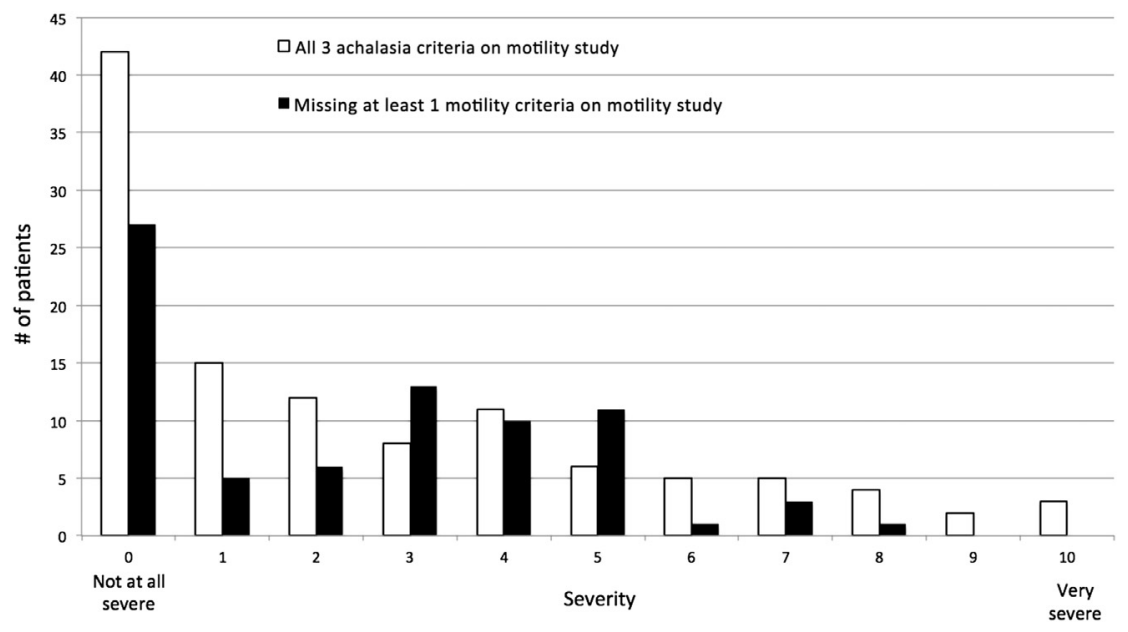

FIGURE 3. Rating of severity of swallowing difficulty according to whether or not all achalasia motility criteria were present on preoperative motility study.

Surgical esophageal myotomy can be accomplished via the chest or abdomen and via an open or minimally invasive thoracoscopic or laparoscopic approach. Although endoscopic pneumatic dilation of LES is the most effective nonsurgical treatment for achalasia, review of patients who underwent surgical myotomy has established surgical myotomy as safe procedure with a superior rate of symptom improvement. ${ }^{2}$ Kostic and colleagues ${ }^{3}$ reported a randomized controlled trial where patients underwent either pneumatic dilation or laparoscopic myotomy. Although only a small number of patients were included in each arm (pneumatic dilation in 26 patients and laparoscopic myotomy in 25 patients), the results showed surgery resulted in better symptom relief and reduced failure rate at 12 months.

In this analysis of our patients we tried to determine the long-term outcome of laparoscopic myotomy. Our median follow-up is almost 6.5 years and like our report on the short-term outcome (5.3 months), the results were quite good. ${ }^{4}$ Almost one-third of patients reported no swallowing problems at all on the follow-up questionnaire and of the remaining that did have trouble, $85 \%$ were less than severe, and almost half had problems only once a week or less. Thus, laparoscopic myotomy when combined with a partial fundoplication seems to be an effective and long-lasting treatment for achalasia. There probably is some deterioration of the efficacy of the myotomy because our results are a bit worse for those with longer follow-up.

Others have found similar outcomes. Weber and colleagues ${ }^{5}$ looked at patients who had a laparoscopic myotomy or pneumatic dilation with at least 5 years of follow-up. They identified 36 studies between 2001 and 2011 and found that for pneumatic dilation, the mean 5 -year remission rate was $61.9 \%$ and the mean 10 -year remission rate was $47.9 \%$. The mean 5- and 10-year remission rates after laparoscopic myotomy were much better at $76.1 \%$ and $79.6 \%$, respectively. They concluded that laparoscopic myotomy affords greater long-term durability.

The report by Boeckxstaens and colleagues ${ }^{6}$ states that results of laparoscopic myotomy are not superior to pneumatic dilation. Our findings do not directly compare the 2 therapeutic modalities, but our follow-up is considerably longer at a median of 6.4 years. As discussed by Patti and Pellegrini, ${ }^{7}$ in the United States most patients are now treated with laparoscopic myotomy with excellent outcomes. We agree with Patti and Pellegrini ${ }^{7}$ that pneumatic dilation should be used for patients that are unfit for definitive laparoscopic surgery.

Patients who present for myotomy without all 3 of the classic manometric findings on preoperative manometry testing pose a difficult management problem. We have shown that even in patients without all the so-called classic findings, results are still good with $30 \%$ having no problems swallowing and only $6.5 \%$ having more severe difficulty at follow-up. It would seem from this data that recommendation of a surgical myotomy is reasonable in patients thought to have achalasia but do not have all 3 of the classic findings on manometry.

Although it is agreed that addition of fundoplication to laparoscopic myotomy decreases gastroesophageal reflux symptoms and acid exposure, there is far less consensus on the type of fundoplication. Most surgeons agree upon a partial fundoplication because a full fundoplication would lead to a high incidence of dysphagia in patients without esophageal peristalsis. We used either an anterior (ie, Dor) or posterior (ie, Toupet) fundoplication in our patients and were not able to detect a difference in the outcome between the 2 techniques. Rawlings and colleagues $^{8}$ also saw this finding of equality between the 
2 different types of partial fundoplication in a randomized trial of the 2 techniques. They carefully studied patients after laparoscopic myotomy with Toupet or Dor fundoplication and found no statistical difference in the dysphagia or regurgitation symptoms. The choice of which technique of fundoplication to use seems to be best left to the surgeon.

A small number of patients $(4.6 \%)$ in our series had a myotomy without an antireflux procedure. More than $80 \%$ of these were performed in the early stages of this series, before the inclusion of an antireflux procedure was appreciated. As noted in the results, these patients were more likely to have reflux symptoms and their symptoms were more frequent. We currently recommend the inclusion of a partial fundoplication for patients undergoing a laparoscopic myotomy.

Esophageal mucosal disruptions occurred in 52 patients $(10.4 \%)$ in our series and all of them were identified intraoperatively and repaired. Rate of intraoperative esophageal perforation during laparoscopic myotomy varies from $0 \%$ to $33 \%$ in several series. ${ }^{2,4,9-17}$ Only 1 patient had a delayed presentation of a mucosal perforation and required a subsequent operation to repair the leak. When mucosal perforations do occur, our current treatment recommendation is that they should be repaired laparoscopically with fine sutures with the addition of a Dor-type fundoplication to buttress the repair. Other than a prior myotomy, prior therapy-such as dilation or botulinum toxin type A-did not seem to affect the rate of esophageal perforation at the time of myotomy. However, nonsurgical procedures, such as dilation or botulinum toxin type A, should not be done as a trial to see if they help, unless the diagnosis is in doubt or the patient is true too ill to undergo surgery. As stated above these nonsurgical therapies are not as effective in the long-term management of achalasia.

The operative morbidity was $3.6 \%$ in our series. Complications in several other series vary from 0 to $16 \%{ }^{9,17}$ The overall complication rate in the review by Campos and colleagues ${ }^{2}$ was $6.3 \%$. Most complications in our series were of minor nature except 2 patients who had bowel perforation and needed subsequent surgery. Similarly, operative mortality has traditionally been low or nonexistent in series involving laparoscopic myotomy., ${ }^{4,14,16,17}$ These results of minimal operative mortality and morbidity reiterate the fact that laparoscopic myotomy is safe. ${ }^{1,18}$

The questionnaire used, the Mayo Dysphagia Questionnaire-30, offers a focused, validated tool for patients with benign esophageal diseases. The Mayo Dysphagia Questionnaire-30 records a patient's dysphagia symptoms at a specific time. The primary use is to assess the frequency and severity of dysphagia and other factors that affect swallowing symptoms. The reliability and concurrent validity of the Mayo Dysphagia
Questionnaire-30 has been established from earlier studies. ${ }^{1,18}$ In our study, we only queried patients at 1 point in time; thus, we may have miscategorized patients with varying symptomatology.

Only $49 \%$ of living patients returned the survey and those who did were closer to their surgical date than the nonresponders (6.4 vs 7.4 years). We hypothesis that the nonresponders may have been less likely to respond because they had become used to any residual symptoms and may have been tired of answering questions about their disease. The long length of the questionnaire ( 7 pages) may have significantly decreased the response rate. Overall the average response rate of mailed questionnaires from physicians is about $60 \% .^{19} \mathrm{We}$ did try to increase our response rate by sending a second mailing to those that did not initially respond and all questionnaires included a return-stamped envelope. If the response rate leads to bias in the outcome is uncertain.

As we demonstrated in our early report, patients who undergo a redo myotomy have a worse outcome when compared with patients undergoing an initial myotomy. ${ }^{4}$ The reasons for this are unclear, but certainly scar tissue and selection bias play a role. We found a similar worse outcome in patients undergoing a redo operation in this series.

Patients older than age 65 years had less likelihood of swallowing problems, heartburn, or regurgitation at the time of follow-up. Roll and colleagues ${ }^{20}$ found similar findings in older patients who underwent laparoscopic myotomy. They found that older patients had better relief of dysphagia, less heartburn, and less chest pain. Also, older patients required less postoperative dilations or additional surgery for recurrent or persistent symptoms. Based on their review, they suggested laparoscopic myotomy as first-line therapy for older patients. Similarly, Gockel and colleagues $^{21}$ found favorable results for patients older than age 40 years and comparable to younger patients.

Although the symptomatic improvement after surgical myotomy is well known in the short- and midterm, our study focused more on the long-term functional outcome after laparoscopic myotomy. At a median follow-up of 77 months we found that a laparoscopic myotomy with partial fundoplication is a durable therapy for patients with achalasia. Older patients tend to have better outcomes at follow-up and should be offered surgical therapy.

\section{CONCLUSIONS}

Laparoscopic myotomy with partial fundoplication is a safe operation. The long-term outcome is extremely effective and approximately one-third of patients who responded to our questionnaire have no evidence of persistent symptoms at follow-up. Furthermore, those who have persistent symptoms rarely have severe or very frequent complaints. Patients older than age 65 years tend to have a better outcome. 


\section{References}

1. McElhiney J, Lohse MR, Arora AS, Peloquin JM, Geno DM, Kuntz MM, et al. The Mayo Dysphagia Questionnaire-30: documentation of reliability and validity of a tool for interventional trials in adults with esophageal disease. Dysphagia. 2010;25:221-30.

2. Campos GM, Vittinghoff E, Rabi C, Takata M, Gadenstatter M, Lin F, et al. Endoscopic and surgical treatments for achalasia: a systematic review and meta-analysis. Ann Surg. 2009;249:45-57.

3. Kostic S, Kjellin A, Ruth M, Lonroth H, Johnsson E, Andersson M, et al. Pneumatic dilatation or laparoscopic cardiomyotomy in the management of newly diagnosed idiopathic achalasia. Results of a randomized controlled trial. World J Surg. 2007;31:470-8.

4. Deb S, Deschamps C, Allen MS, Nichols FC III, Cassivi SD, Crownhart BS, et al. Laparoscopic esophageal myotomy for achalasia: factors affecting functional results. Ann Thorac Surg. 2005;80:1191-4; discussion 1194-5.

5. Weber CE, Davis CS, Kramer HJ, Gibbs JT, Toles L, Fisichella PM. Medium and long-term outcomes after pneumatic dilation or laparoscopic Heller myotomy for achalasia: a meta-analysis. Surg Laparosc Endosc Percutan Tech. 2012;22: 289-96.

6. Boeckxstaens GE, Annesse V, des Varannes SB, Chaussade S, Constantini M, Cuttitta A, et al. Pneumatic dilation versus laparoscopic Heller's myotomy for idiopathic achalasia. N Engl J Med. 2011;364:1807-16.

7. Patti MG, Pellegrini CA. Esophageal achalasia 2011: pneumatic dilatation or laparoscopic myotomy? J Gastrointest Surg. 2012;16:870-3.

8. Rawlings A, Soper NJ, Oelschlager B, Swanstrom L, Matthews BD, Pellegrini C, et al. Laparoscopic Dor versus Toupet fundoplication following Heller myotomy for achalasia: results of a multicenter, prospective, randomized-controlled trial. Surgical Endosc. 2012;26:18-26.

9. Avtan L, Avci C, Guvenc H, Igci A, Ozmen V. Laparoscopic myotomy for oesophageal achalasia-adding an antireflux procedure is not always necessary. Int J Clin Pract. 2005;59:35-8.

10. Ramacciato G, D’Angelo FA, Aurello P, Del Gaudio M, Varotti G, Mercantini P, et al. Laparoscopic Heller myotomy with or without partial fundoplication: a matter of debate. World J Gastroenterol. 2005;11:1558-61.

11. Rossetti G, Brusciano L, Amato G, Maffettone V, Napolitano V, Russo F, et al. A total fundoplication is not an obstacle to esophageal emptying after heller myotomy for achalasia: results of a long-term follow up. Ann Surg. 2005;241: 614-21.

12. Bonatti H, Hinder RA, Klocker J, Neuhauser B, Klaus A, Achem SR, et al. Long-term results of laparoscopic Heller myotomy with partial fundoplication for the treatment of achalasia. Am J Surg. 2005;190:874-8.

13. Rosemurgy A, Villadolid D, Thometz D, Kalipersad C, Rakita S, Albrink M, et al. Laparoscopic Heller myotomy provides durable relief from achalasia and salvages failures after botox or dilation. Ann Surg. 2005;241:725-33; discussion $733-5$.

14. Khajanchee YS, Kanneganti S, Leatherwood AE, Hansen PD, Swanstrom LL. Laparoscopic Heller myotomy with Toupet fundoplication: outcomes predictors in 121 consecutive patients. Arch Surg. 2005;140:827-33; discussion 833-4.

15. Burpee SE, Mamazza J, Schlachta CM, Bendavid Y, Lein L, Moloo H, et al. Objective analysis of gastroesophageal reflux after laparoscopic heller myotomy: an anti-reflux procedure is required. Surgical Endosc. 2005;19: 9-14.

16. Katada N, Sakuramoto S, Kobayashi N, Futawatari N, Kuroyama S, Kikuchi S, et al. Laparoscopic Heller myotomy with Toupet fundoplication for achalasia straightens the esophagus and relieves dysphagia. Am J Surg. 2006; 192:1-8.

17. Torquati A, Richasrds WO, Holzman MD, Sharp KW. Laparoscopic myotomy for achalasia: predictors of successful outcome after 200 cases. Ann Surg. 2006;243:587-91; discussion 591-3.

18. Grudell AB, Alexander JA, Enders FB, Pacifico R, Fredericksen M, Wise JL. Validation of the Mayo Dysphagia Questionnaire. Dis Esophagus. 2007;20: $202-5$.

19. Cummings SM, Savitz LA, Konrad TR. Reported response rates to mailed physician questionnaires. Health Serv Res. 2001;35:1347-55.

20. Roll GR, Ma S, Gasper WJ, Patti M, Way LW, Carter J. Excellent outcomes of laparoscopic esophagomyotomy for achalasia in patients older than 60 years of age. Surg Endosc. 2010;24:2562-6.

21. Gockel I, Gith A, Drescher D, Jungmann F, Eckhard L, Lang H. Minimally invasive surgery for achalasia in patients $>40$ years: more favorable than anticipated. Langenbeck's Arch Surg. 2012;397:69-74.

\section{Discussion}

Dr Bryan F. Meyers (St Louis, Mo). Congratulations on your presentation. This is a big study with consistent techniques. You have excellent clinical work and no deaths out of 500 patients, not even a rare pulmonary embolism or some other freak complication. It was near-perfect initial results. You had only 1 late leak at the myotomy site, which is excellent. I commend you on the notion of intent to use a standardized technique to measure a subjective outcome that can be very difficult to pin down.

I do have to mention a few criticisms. There is no preoperative use of the Mayo Dysphagia Questionnaire-30. Your tool was not used preoperatively, so it limits your ability to show an improvement or the degree of improvement in any of the patients or look for associations between preoperative factors and the degree of improvement. There is no discussion of additional surgery over the course of their recovery. So a redo myotomy, subsequent esophagectomy, or the occasional hiatal hernia that happens after a Dor are not addressed. What role they played on patients' quality of life are not touched, and to that extent it doesn't really predict the experience or outcome of the patients. You have missing data in $50 \%$ of patients, and what happened to the missing patients could really dictate the overall group when half didn't return the questionnaires.

I have 3 very brief and focused questions. Did you use the same validated tool throughout? Your first patient was in 1998, but the 2 references on the tool were in 2007 and 2010, and so there is a big lag time there.

Dr Krishnamohan. The same questionnaire was used for all patients in the study.

Dr Meyers. With the missing data, can you really infer that older patients do better? You didn't really assess them before, and I could give you several plausible explanations why they have fewer complaints later: they have other medical problems that trump their swallowing or they have poor memory, so they can't remember 30 days ago when they choked on steak. Can you give me a biologic explanation of why they would do better?

Dr Krishnamohan. I appreciate that point. It would be good if we had a questionnaire assessment at several points of time for those patients or another timed barium swallow to add additional information. This questionnaire has been validated in other studies to assess benign esophageal symptoms, and we expect that patients who answer within the 30 days should be reflective, but there could be a bias. Other studies have shown that the average response rate for any questionnaire sent by a physician averages around $60 \%$, and our response rate was $48 \%$. We tried to improve the response rate by sending a second questionnaire, but the best response we could get was $48 \%$.

Dr Meyers. I know it is a challenge, but it's hard to know what to do when half the patients don't respond. Half could be windsurfing in Florida or half might be in a nursing home aspirating. We just don't know what is going on with them. We hope the ones you got are representative of all of them, but, to a large extent, we don't know. Anyway, this is excellent, qualitative work that helps generate hypotheses and maybe lays a foundation for future work, but it is hard to come up with a solid change in practice based on qualitative work with half the patients not responding. 
Dr Krishnamohan. Thank you for your remarks. When we looked at the patients who responded versus those who did not respond, the only difference between the 2 groups we could find was the length of follow-up. Patients who responded-the median-they were 6.5 years from surgery, when those who did not respond were farther out from surgery-7.5 years-and the difference was significant, but other parameters were not different between the 2 groups.

Dr Mark K. Ferguson (Chicago, Ill). I enjoyed your presentation, thank you.

I have 2 pretty simple questions. First, in my practice we rarely use manometry to make the diagnosis of achalasia. It is pretty simple to make a clinical diagnosis with a barium swallow and then rule out other problems with endoscopy. I'm wondering how your institution uses the information from manometry to either select patients for surgery or tailor the operation differently than it might be done in a routine fashion.

Dr Krishnamohan. The manometry helps to confirm the diagnosis and occasionally will lead us to perform a different type of operation.

Dr Ferguson. The second question is about the terminology used in your questionnaire. You speak in your presentation about swallowing difficulty. I'm not sure if that is the exact terminology that is used in the questionnaire. When I ask patients about difficulty swallowing, at least a third of them will say, "No, I have no problems swallowing, but then the food sticks down here afterward." So when I ask the question, it doesn't really get at the severity of symptoms that they are having from achalasia.

Dr Krishnamohan. The questionnaire has 28 questions and many subquestions. It includes symptoms about heartburn and regurgitation, including the severity and frequency. The questionnaire is a very complete assessment.

Dr Ferguson. So you are speaking of swallowing difficulty as a composite score of a complex questionnaire.

Dr Krishnamohan. Yes, we tried to report the results of the questionnaire to reflect the overall outcome.

Dr Ferguson. Thank you.

Dr Steven R. DeMeester (Los Angeles, Calif). Congratulations on a large series and a nice presentation.

One of the long-term problems with treatment of achalasia is reflux disease and the complications of reflux disease on an aperistaltic esophagus. I was struck by the fact that $70 \%$ of your patients had trouble swallowing and $70 \%$ also had heartburn or reflux symptoms. Do you have an idea if the difficulty with swallowing was a complication of reflux or was this an incomplete myotomy? And the reflux really needs to be explored. That's one of the concerns we have with the per-oral endoscopic myotomy procedure - we're not adding an antireflux procedure to it-and long term what are the implications of that? I encourage you to really look at that reflux and assess how severe the reflux disease was and if there were strictures from reflux that caused the trouble swallowing.
Dr Krishnamohan. We performed a partial fundoplication as routine for most patients. The set of patients who did not have a fundoplication were earlier on in the series when the importance of fundoplication was not really established and it was controversial. There have been other studies that show that the swallowing disorder after a fundoplication is comparable to those patients who did not have a fundoplication, but the incidence of pathologic and clinical gastroesophageal reflux is lower if there is a fundoplication with a myotomy.

Dr Thomas Rice (Cleveland, Ohio). I have some comments.

Achalasia is a neurodestructive, lifelong disease, so I object to a long-term follow-up of 28 days. I think it is very, very short early postoperative follow-up that you have done. Also, in your definition of achalasia, and the requirement of a hypertensive lower esophageal sphincter is very, very uncommon. Fewer than one-third of people have it. As well, you defined a hypertensive lower esophageal sphincter as $>24 \mathrm{~mm} \mathrm{Hg}$, but normal resting pressure is up to $40 \mathrm{~mm} \mathrm{Hg}$. Depending upon the manometry, that is considered normal.

Just a few comments and about manometry. Achalasia is a manometrically defined disease, so it is required in all patients and I would strive to do high-resolution manometry in all patients so that they have best diagnostic and follow-up studies.

There is a big disconnect between symptoms, function, and morphology, and, really, to do a timed barium esophagram and high-resolution manometry postoperatively would be helpful and supplement the symptom score.

Dr Tomasz Grodzki (Szczecin, Poland). I have a short question. The major complaint of the patients was lack of peristalsis. Did you give them any medication to improve it? The results that one-third of patients improved does not sound so optimistic. You did a physical job in experienced hands and the improvement was not so satisfactory. Did you try any complex therapy, or it was just surgery and nothing more?

Dr Krishnamohan. I think you have misunderstood our results. We showed that one-third of patients had no symptoms at all, no problems swallowing, and those who had some residual symptoms for the most part had only mild symptoms.

Dr Frank D'Ovidio (New York, NY). Congratulations on your nice presentation.

If you were able to subgroup patients according to type of achalasia-for example, patients with preserved body of the esophagus, those with mega esophagus, those with a sigmoid esophagus, and also patients with spastic achalasia-would these different forms of achalasia have different preoperative and postoperative presentations and also long-term results?

Dr Krishnamohan. We did not try to differential patients based on those subgroups. We reviewed retrospectively all patients who underwent a myotomy. I'm sure this group of 500 did have all those types of achalasia but we did not attempt to subclassify those patients or study the results. 